\title{
Sesquiterpenes $\alpha$-humulene and $\beta$-caryophyllene oxide enhance the efficacy of 5-fluorouracil and oxaliplatin in colon cancer cells
}

\author{
MARTIN AMBROŽ \\ MARKÉTA ŠMATOVÁ ${ }^{1}$ \\ MICHAELA ŠADIBOLOVÁ ${ }^{1}$ \\ EVA POSPÍŠILOVÁ ${ }^{1}$ \\ PAVLÍNA HADRAVSKÁ ${ }^{1}$ \\ MICHAELA KAŠPAROVÁ ${ }^{1}$ \\ VERONIKA HANUŠOVÁ SKARKOVÁ \\ VĚRA KRÁLOVÁ ${ }^{2}$ \\ LENKA SKÁLOVÁ ${ }^{1 *}$ \\ ${ }^{1}$ Department of Biochemical Sciences \\ Charles University in Prague \\ Faculty of Pharmacy, Heyrovského \\ 1203, Hradec Králové, CZ-500 05 \\ Czech Republic \\ ${ }^{2}$ Department of Medical Biology and \\ Genetics, Charles University in Prague \\ Faculty of Medicine in Hradec Králové \\ Šimkova 870, Hradec Králové \\ CZ-500 38, Czech Republic
}

\begin{abstract}
The present study is designed to find out if sesquiterpenes, $\alpha$-humulene (HUM), valencene (VAL), $\beta$-caryphylleneoxide (CAO) and trans-nerolidol (NER), are able to improve the antiproliferative effect of classical cytostatic drugs, 5-fluorouracil (FU) and oxaliplatin (1,2-diaminocyclohexaneoxalato-platinum, $\mathrm{OxPt}$ ), in colon cancer cell lines Caco-2 and SW-620. In addition, the possible mechanisms of sesquiterpene action are studied. The results show significant ability of HUM and especially of CAO to enhance the antiproliferative effects of $\mathrm{FU}$ and $\mathrm{OxPt}$ in cancer cell lines Caco-2 and SW-620. On the other hand, VAL and NER are ineffective. The action of CAO could be partly based on its ability to disrupt the mitochondrial membrane potential and to activate initiator caspases, but other mechanisms are probably also involved. Based on these results, CAO seems to have the potential for combination therapy of colon cancers and deserves further study.
\end{abstract}

Keywords: terpenes, Caco-2, SW-620, combination therapy

Accepted July 17, 2018

Published online August 29, 2018

Despite advances in diagnostics, surgery and targeted therapy, colorectal cancers have very high prevalence and mortality, and thus the search for new therapeutic possibilities is of great importance (1). Plants are rich reservoirs of secondary metabolites with anti-cancer activities. Especially essential oils and their constituents, terpenes, could be proper candidates (2).

Essential oil from the leaves of Myrica rubra (Sieb. et Zucc, Myricaceae, MEO), traditionally used in folk medicine, exhibited significant antiproliferative activity in several intestinal cancer cell lines (3). GC $\times$ GC/TOF-MS analysis of MEO identified sesquiterpenes $\beta$-caryophyllene, $\alpha$-humulene (HUM), humulene epoxide I, valencene (VAL), epi- $\alpha$ selinene, $\gamma$-murolene, $\beta$-caryophyllene-oxide (CAO) and trans-nerolidol (NER) as major compounds (3). In the following studies, antiproliferative and pro-oxidative effects of HUM, VAL, CAO and NER were shown in intestinal, ovarian and lymphoblast cancer cell

\footnotetext{
*Correspondence; e-mail: lenka.skalova@faf.cuni.cz
} 
lines $(4,5)$. Many other sesquiterpenes possess anticancer activities, mainly based on their ability to activate apoptosis, to arrest cell cycle, or to inhibit proangiogenic, invasive and proliferative markers (2). In addition, some sesquiterpenes were able to improve the efficacy of classical anticancer drugs when used in combinations. For example, $\beta$-caryophyllene increased antiproliferative activity of paclitaxel and its intracellular accumulation in several cancer cell lines (6). Also CAO improved the efficacy of paclitaxel and doxorubicin in human myeloma and prostate cancer cells (7).

In our previous studies, MEO as well as its components CAO, HUM, NER and VAL were tested in view of their possible ability to improve the efficacy of doxorubicin (DOX) in various cancer cell lines (8). In colon cancer cells Caco-2, MEO enhanced the DOX antiproliferative and pro-oxidative effect and increased DOX intracellular concentration. CAO, NER and VAL significantly enhanced antiproliferative efficacy of DOX in Caco- 2 cells. All sesquiterpenes were able to increase DOX accumulation selectively in cancer cells. In addition, CAO and VAL were able to increase the DOX pro-oxidative effect (4). In DOX-sensitive lymphoblast CCFR/CEM cells, synergism of DOX with CAO and NER was observed. On the other hand, sesquiterpenes did not increase DOX efficacy in DOX-resistant CEM/ ADR cells, although they significantly increased accumulation of DOX and rhodamine-123 (substrate of efflux transporter ABCB1) within cancer cells. A strong synergism of DOX with NER, HUM and CAO was found in ovarian cancer cells (5).

Taken together, the results mentioned above show that specific sesquiterpenes from MEO have the potential to enhance DOX efficacy in anticancer therapy. The action of these sesquiterpenes is based (at least partly) on their ability to increase oxidative stress in cancer cells. However, we would like to clarify the mechanism of the pro-oxidative effect of these sesquiterpenes and their ability to improve the efficacy of other anticancer drugs used in colon cancer therapy. For this reason, the present study was designed to evaluate, for the first time, the effects of sesquiterpenes HUM, CAO, NER and VAL on the mitochondrial membrane potential and their influence on the efficacy of FU and OxPt in colon adenocarcinoma cells. OxPt, a third-generation platinum compound, is widely used in gastrointestinal malignancies. Its main cellular target is DNA, where it forms cross-links. FU, a prominent cytostatic for several cancers including colon cancer, is a pyrimidine analog that acts as an antimetabolite stopping the synthesis of DNA.

In addition, the most effective sesquiterpene is also studied in view of its possible ability to increase the activities of caspases. Cell line CaCo-2 was used since MEO has exerted the highest efficacy against these colon adenocarcinoma cells (3). In the combination study, the cell line SW-620 is also used as an aggressive colon cancer cell, derived from the metastatic site.

\section{EXPERIMENTAL}

\section{Chemicals and reagents}

Individual sesquiterpenes, $\mathrm{FU}, \mathrm{OxPt}$, carbonyl cyanide 3-chlorophenylhydrazone (CCCP) (all at the highest purity available), Eagle's minimum essential medium (EMEM), Dulbecco's modified Eagle's medium (DMEM), N-2-hydroxyethylpiperazine- $N$ '-2ethanesulfonic acid (HEPES) buffer, MEM non-essential amino acid solution, Hank's balanced salt solution, 5,5',6,6'-tetrachloro-1,1',3,3'-tetraethylbenzimidazolylcarbocyanine io- 
dide (JC1 dye), 3-[(3-cholamidopropyl)dimethylammonio]-1-propanesulfonate (CHAPS), dithiothreitol (DTT), dimethylsulphoxide (DMSO), penicillin, streptomycin, L-glutamine and neutral red were supplied by Sigma-Aldrich (USA). Fetal bovine serum (FBS) and gentamicin sulfate were purchased from Invitrogen (UK) and bovine serum albumin (BSA) from Fluka, Switzerland. Stock solutions were prepared in dimethyl sulphoxide and stored at $4{ }^{\circ} \mathrm{C}$ in the dark.

\section{Cell cultures}

Human epithelial colorectal adenocarcinoma lines CaCo-2 and SW-620 were purchased from the American Type Culture Collection (ATCC, Manassas, USA). Cells were multiplied in three passages, frozen in aliquots and stored in liquid nitrogen. The absence of mycoplasma in all cell lines used in the laboratory was periodically checked by commercial analysis in Generi Biotech (Czech Republic). For every set of experiments (lasting 3-9 weeks), new storage cells were resuscitated. The CaCo-2 cells were cultured in EMEM supplemented with $10 \%$ heat-inactivated FBS, $1 \%$ MEM non-essential amino-acid, $1.5 \%$ L-glutamine solution and $0.5 \%$ penicillin/streptomycin. Human metastatic colon adenocarcinoma SW-620 cells were maintained in DMEM medium supplemented with $10 \%$ heat-inactivated FBS and $1 \%$ penicillin/streptomycin. Cells were grown in T-75 $\mathrm{cm}^{2} \mathrm{cul}-$ ture flasks in a humidified atmosphere containing $5 \% \mathrm{CO}_{2}$ at $37^{\circ} \mathrm{C}$.

\section{Tests of cell proliferation}

Sesquiterpenes were pre-dissolved in DMSO; the concentration of DMSO in medium was $0.1 \%$. FU and OxPt were pre-dissolved in redistilled water. Cells were cultured in 96-well plates and exposed to various concentrations of the tested compounds in culture medium for 72 hours. In combination tests, concentrations of both substances were chosen according to their $I C_{50}$ values and the tested concentrations represented the multiples of these values at a constant ratio. Cells cultured in medium with $0.1 \%$ DMSO without tested compounds were used as control cells. Cells cultured in medium with $10 \%$ DMSO (completely destroyed cells) served as a positive control. The number of viable cells was assayed using the neutral red uptake assay (NRU) as described previously (5).

\section{Activity of caspases}

Cells were cultured in 96-well plates for 48 hours. The medium was then removed and fresh medium with tested compounds was added. After 4, 8, 24-hour exposition, lysis buffer $\left(50 \mathrm{mmol} \mathrm{L}^{-1}\right.$ hydroxyethylpiperazine- $N^{\prime}$-2-ethanesulfonic acid, $5 \mathrm{mmol} \mathrm{L}{ }^{-1} 3$-[(3-cholamidopropyl)dimethylammonio]-1-propanesulfonate, $5 \mathrm{mmol} \mathrm{L}^{-1}$ dithiothreitol, $\mathrm{pH} 7.4$ ) was added. Cell lysates were frozen and stored in a freezer $\left(-80^{\circ} \mathrm{C}\right)$ until analysis. Before measurement, the samples were thawed. Caspases activities were measured using the caspase-GLO assay (Promega, USA) according to the manufacturer's manual.

\section{Mitochondrial membrane potential}

Cells were cultured in 24-well plates for 24 hours. Then, JC1 dye (final concentration $5 \mu \mathrm{mol} \mathrm{L}^{-1}$ ) was added to the medium. After 30-min incubation, the cells were washed with 
Hank's balanced salt solution (HBSS). Tested sesquiterpenes and carbonyl cyanide 3-chlorophenylhydrazone (CCCP) stock solutions, diluted in HBSS, were added to the cells. Three concentrations $\left(50,100,150 \mu \mathrm{mol} \mathrm{L}^{-1}\right)$ of sesquiterpenes and three incubation times (1, 2 and $4 \mathrm{~h}$ ) were used. The CCCP served as a positive control. After incubation, the fluorescence was measured and the ratio of fluorescence $(590 / 530 \mathrm{~nm})$ was calculated.

\section{Statistical analysis}

The GraphPad Prism 6.0 statistical software was used with statistical significance at $p<0.05$.

\section{RESULTS AND DISCUSSION}

Several mechanisms of anti-cancer activity of sesquiterpenes were considered. One could be based on sesquiterpene ability to increase oxidative stress in cancer cells (2). For example, bigelovin, a sesquiterpene lactone isolated from Inula helianthus aquatica, has been

a)

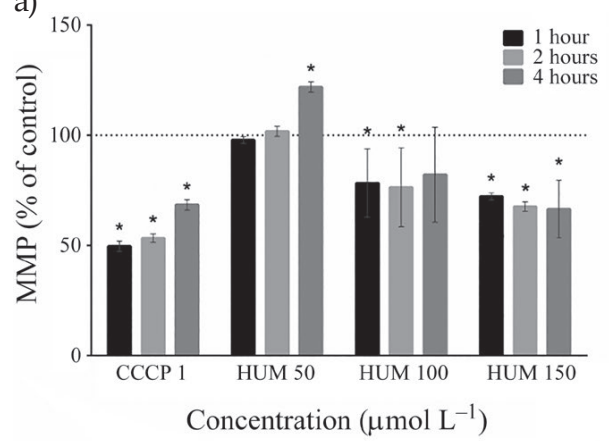

c)

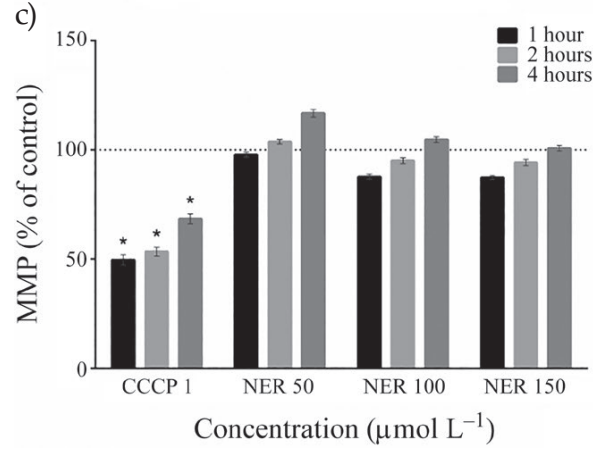

b)

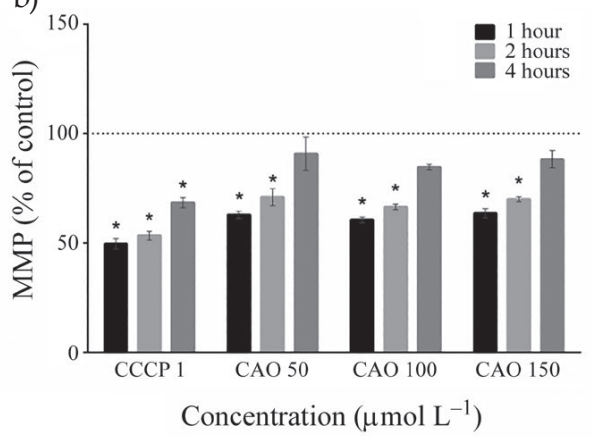

d)

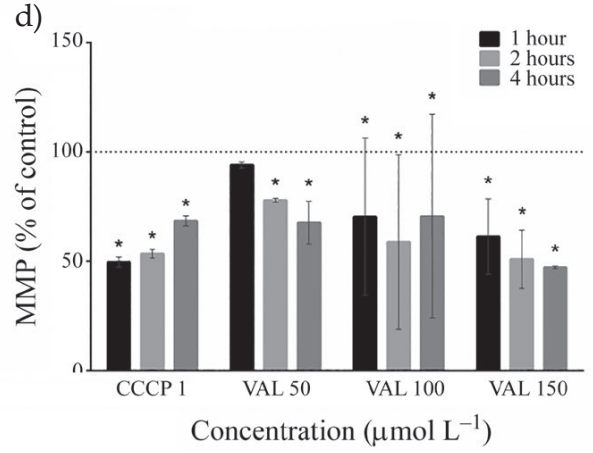

Fig. 1. Effects of sesquiterpenes: a) $\alpha$-humulene (HUM), b) $\beta$-caryophyllene oxide (CAO), c) nerolidol (NER) and d) valencene (VAL) on mitochondrial membrane potential (MMP) in Caco-2 cells tested using the JC-1 assay. Data (mean $\pm \mathrm{SD}$ ) are expressed as percentage of MMP in untreated cells (control: $100 \%$ ). Significant decrease $v$ s. control: ${ }^{*} p<0.05$. 
M. Ambrož et al.: Sesquiterpenes $\alpha$-humulene and $\beta$-caryophyllene oxide enhance the efficacy of 5-fluorouracil and oxaliplatin in colon cancer cells, Acta Pharm. 69 (2019) 121-128.

proven to suppress cell proliferation and induce apoptosis in human colorectal cancer HT29 and HCT 116 cells via G2/M cell cycle arrest, DNA damage and increase in reactive oxygen species (ROS) (9). Treatment of bladder cancer T24 cells with costunolide resulted in a dose-dependent inhibition of cell viability and induction of apoptosis, which was associated with ROS generation (10). In our previous study, the effect of selected sesquiterpenes from MEO on ROS formation was tested in Caco-2 cells (4). CAO and VAL had significant pro-oxidative effects, while HUM did not influence ROS formation and NER acted as a strong antioxidant. Taking in consideration the structure of CAO and VAL, direct ROS generation was unlikely, but there are other mechanisms how sesquiterpenes can induce ROS formation (2). One possible mechanism could be based on sesquiterpene-mediated disruption of the mitochondrial membrane.

Therefore, the influence of CAO, HUM, NER and VAL on the mitochondrial membrane potential (MMP) was tested in Caco-2 cells using the JC-1 assay. Three concentrations $\left(50,100,150 \mu \mathrm{mol} \mathrm{L}^{-1}\right)$ of sesquiterpenes and three incubation times (1, 2 and 4 hours) were used. The carbonyl cyanide 3-chlorophenylhydrazone (CCCP) served as a positive control. The results are demonstrated in Fig. 1.

HUM in $50 \mu \mathrm{mol} \mathrm{L}{ }^{-1}$ concentration did not affect MMP, while $150 \mu \mathrm{mol} \mathrm{L}-1$ HUM significantly decreased MMP after all incubation times. CAO in all concentrations significantly decreased MMP after 1- and 2-h incubation, but its effect was no longer significant after 4-h incubation. Incubation of Caco-2 cells with VAL in all concentrations caused a significant decrease of MMP. Interestingly, the effect of VAL was stronger after 4-h than after 1-h incubation compared to CCCP (positive control) and CAO. NER did not significantly disrupt MMP in Caco-2 cells. In the case of CAO and CCCP, longer exposure led surprisingly to milder effects. It could indicate that cells are able to deactivate quickly both substances (maybe via biotransformation) and to repair damaged membranes. Based on these results, we assume that the pro-oxidative effect of CAO and VAL is mediated through disruption of mitochondrial membranes. The ability of some other sesquiterpenes to decrease MMP has been reported as well (11). For example, cordycepol C triggered a loss of MMP in HepG2 cells in a time- and dose-dependent manner.

The main aim of this project was to find out if sesquiterpenes CAO, HUM, VAL and NER are able to improve the efficacy of classical cytostatics in colon cancer cells. In our previous studies, these sesquiterpenes enhanced the efficacy of the anticancer drug DOX in several colon cancer cell lines (4). The present study is designed to evaluate the ability of these sesquiterpenes to improve the efficacy of oxaliplatin and 5-fluorouracil, two other drugs commonly used in colon cancer cell therapy. The antiproliferative effect of OxPt and FU alone and in combinations with individual sesquiterpenes is tested in Caco-2 and SW620 cell lines. Experiments are designed according to the recommendation of Chou-Talalay (12). The results obtained in Caco-2 cells are presented in Fig. 2.

At higher concentrations, HUM, CAO and VAL in combinations were able to improve the efficacy of OxPt in Caco-2 cells compared to OxPt alone. HUM increased OxPt efficacy also at lower concentrations, while NER did not affect OxPt efficacy in Caco-2 cells at any concentration. FU combinations with $\mathrm{CAO}$ showed a stronger antiproliferative effect at all concentrations compared to FU alone. All other sesquiterpenes improved FU efficacy in Caco-2 cells, but only at the highest concentrations. Moreover, NER and VAL at lower concentrations even impaired FU efficacy. 
a)

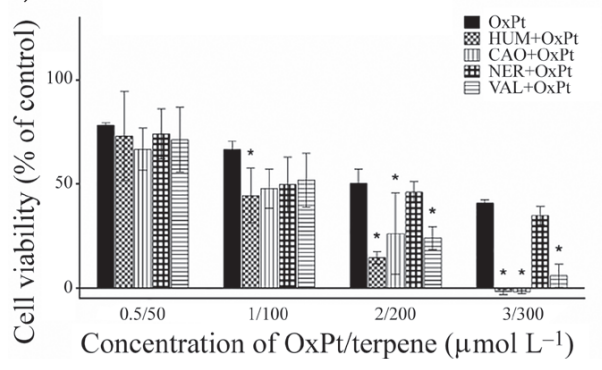

b)

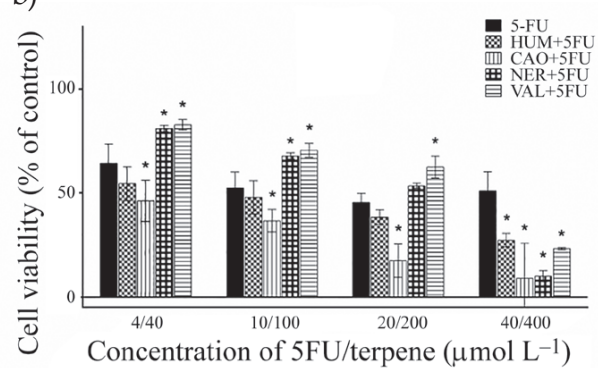

Fig. 2. The antiproliferative effect of: a) oxaliplatin (OxPt) and b) 5-fluorouracil (FU) alone and in combinations with individual sesquiterpenes in Caco-2 cell line. Data (mean $\pm \mathrm{SD}$ ) are expressed as percentage of viability of untreated cells (control: $100 \%$ ). Significant increase/decrease vs. control: ${ }^{*} p<0.05$.

a)

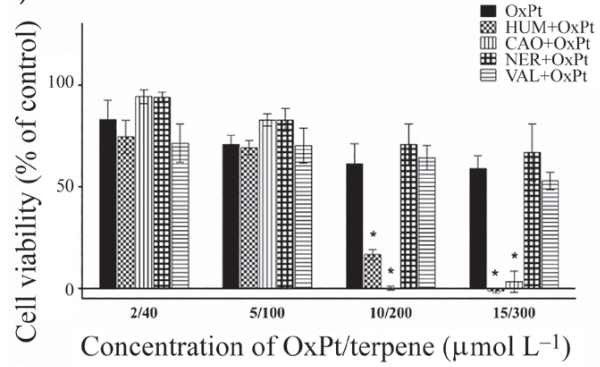

b)

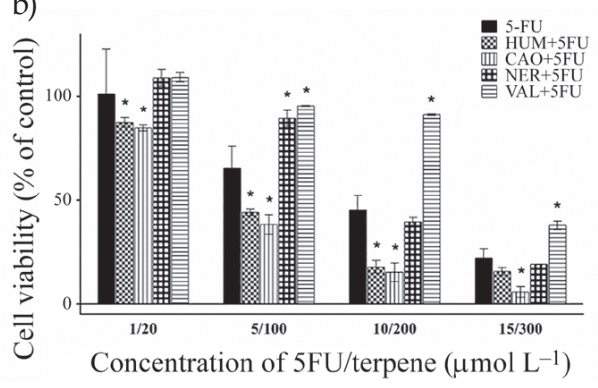

Fig. 3. The antiproliferative effect of: a) oxaliplatin (OxPt) and b) 5-fluorouracil (FU) alone and in combinations with individual sesquiterpenes in SW-620 cell line. Data (mean \pm SD) are expressed as percentage of viability of untreated cells (control: $100 \%$ ). Significant increase/decrease $v$ s. control: ${ }^{*} p<0.05$.

a)

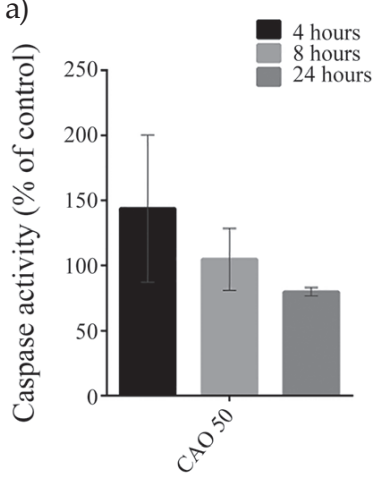

b)

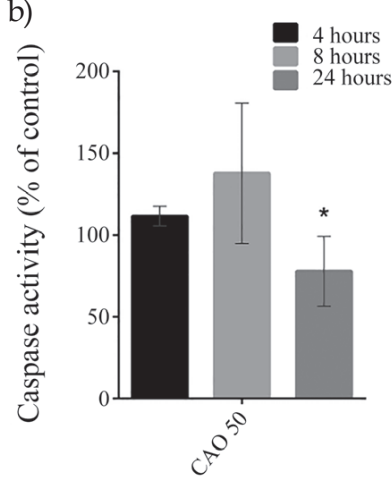

c)

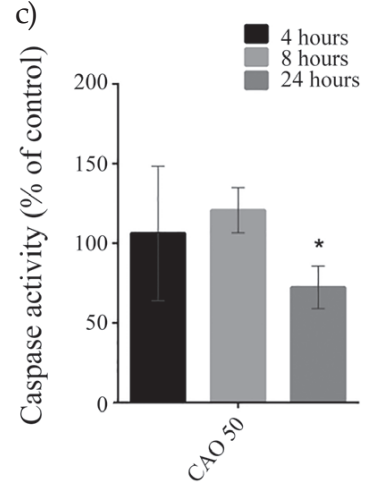

Fig. 4. The effect of $\beta$-caryophyllene oxide (CAO) on the activities of: a) caspases 3/7, b) caspase 8 and c) caspase 9 in Caco-2 cells, after 4-, 8- and 24-h exposition. Data (mean \pm SD) are expressed as percentage of viability of untreated cells (control: $100 \%$ ). Significant increase/decrease vs. control: ${ }^{*} p<0.05$. 
M. Ambrož et al.: Sesquiterpenes $\alpha$-humulene and $\beta$-caryophyllene oxide enhance the efficacy of 5-fluorouracil and oxaliplatin in colon cancer cells, Acta Pharm. 69 (2019) 121-128.

As regards SW-620 cells, OxPt in combinations with HUM and CAO had a stronger antiproliferative effect than OxPt alone, but NER and VAL were not able to improve $\mathrm{OxPt}$ efficacy in these cells (see Fig. 3a). HUM and CAO also potentiated the antiproliferative effect of FU in SW-620 cells. On the other hand, combinations of FU with VAL (at all concentrations) and FU with NER (at one concentration) had a weaker antiproliferative effect than FU alone (see Fig. 3b).

Taken together, sesquiterpenes CAO and HUM significantly enhanced the efficacy of $\mathrm{OxPt}$ and FU in colon cancer cells Caco-2. Moreover, these sesquiterpenes were able to improve OxPt and FU efficacy in metastatic cancer cells SW-620. These results are promising in view of the potential use of CAO and HUM in combination therapy of colon cancers. In our previous studies, CAO increased the efficacy of DOX in cancer colon cells Caco-2, lymphoblast cells CCFR/CEM, and two cancer ovarian cell lines, while HUM was able to enhance DOX efficacy only in ovarian cancer cells $(4,5)$. On the other hand, NER also improved DOX efficacy in all the cell lines mentioned above, but it did not enhance the efficacy of FU and OxPt in colon cancer cell lines Caco-2 and SW-620. These results show that the ability of individual sesquiterpenes to improve the efficacy of cytostatics significantly differs in dependence on cancer cells and the drug used.

Nevertheless, CAO seems to be the most promising and hence it was chosen for further experiments. To find out if combinations of $\mathrm{CAO}$ with $\mathrm{FU}$ or OxPt directed cancer cells into apoptosis, the effect of $\mathrm{CAO}, \mathrm{FU}$ and $\mathrm{OxPt}$ alone, and $\mathrm{CAO}+\mathrm{FU}, \mathrm{CAO}+\mathrm{OxPt}$ combinations on the activity of the initiating caspases 8 and 9 and also on the effector caspases 3/7 activity was tested in the Caco-2 cell line (Fig. 4). The activity of selected caspases was evaluated after 4-, 8- and 24-h exposure. Incubation of Caco-2 cells with CAO alone caused no elevation of caspase activity; caspase 8 was even mildly decreased after 24-hour CAO treatment. This finding indicates that $\mathrm{CAO}$ action is not caspase-dependent. Another sesquiterpene, cordycepol C, did not increase caspase activities either, although it showed a significant antiproliferative effect in HepG2 cells (11). On the other hand, telekin induced activation of caspase 9 and caspase 3 in HepG-2 cells (13).

In our study, neither FU nor OxPt in concentrations used increased caspase 8 activity. On the other hand, when Caco-2 cells were treated for 8 hours with CAO and FU in combination, the activities of both caspases 8 and 9 increased. The combination of CAO with OxPt caused an increase of only caspase 9 activities compared to FU or OxPt alone.

\section{CONCLUSIONS}

Our results indicate, for the first time, that HUM and especially CAO have significant potential to enhance FU- and OxPt-mediated cancer cell killing. The ability of CAO to disrupt mitochondrial membranes (leading to possible release of cytochrome $\mathrm{c}$ and to an increase of oxidative stress) could participate in its action, but further mechanisms are probably involved. In conclusion, $\mathrm{CAO}$ seems to be the most promising in the enhancement of FU and OxPt efficacy in colon cancer cells and it deserves further study.

Acknowledgements. - This work was funded by the Czech Science Foundation, Centre of Excellence, No. P303/12/G163 and by Charles University in Prague, research project SVV 260416.

Abbreviations, acronyms, symbols. - ABCB1 - ATP-binding cassette transporter B1, ATP - adenosine triphosphate, $\mathrm{CAO}$ - caryophyllene oxide, $\mathrm{CCCP}$ - carbonyl cyanide 3-chlorophenylhydrazone, CHAPS - 3-[(3-cholamidopropyl)dimethylammonio]-1-propanesulfonate, DMEM - Dulbecco's mod- 
M. Ambrož et al.: Sesquiterpenes $\alpha$-humulene and $\beta$-caryophyllene oxide enhance the efficacy of 5 -fluorouracil and oxaliplatin in colon cancer cells, Acta Pharm. 69 (2019) 121-128.

ified Eagle's medium, DOX - doxorubicin, DTT - dithiothreitol, EMEM - Eagle's minimum essential medium, FBS - fetal bovine serum, FU - 5-fluorouracil, HEPES - hydroxyethylpiperazine- $N^{\prime}-2-$ ethanesulfonic acid, HUM - humulene, MEO - essential oil from Myrica rubra leaves, MMP - mitochondrial membrane potential, NER - trans-nerolidol, OxPt - oxaliplatin, VAL - valencene.

\section{REFERENCES}

1. W. A. Hammond, A. Swaika and K. Mody, Pharmacologic resistance in colorectal cancer: a review, Ther. Adv. Med. Oncol. 8 (2016) 57-84; https://doi.org/10.1177/1758834015614530

2. H. Bartikova, V. Hanusova, L. Skalova, M. Ambroz and I. Bousova, Antioxidant, pro-oxidant and other biological activities of sesquiterpenes, Curr. Top. Med. Chem. 14 (2014) 2478-2494.

3. L. Langhansova, V. Hanusova, J. Režek, B. Stohanslova, M. Ambroz, V. Kralova, T. Vanek, J. D. Lou, Z. L. Yun, J. Yang and L. Skálova, Essential oil from Myrica rubra leaves inhibits cancer cell proliferation and induces apoptosis in several human intestinal lines, Ind. Crops Prod. 59 (2014) 20-26; https://doi.org/10.1016/j.indcrop.2014.04.018

4. M. Ambroz, I. Bousova, A. Skarka, V. Hanusova, V. Kralova, P. Matouskova, B. Szotakova and L. Skalova, The influence of sesquiterpenes from Myrica rubra on the antiproliferative and pro-oxidative effects of doxorubicin and its accumulation in cancer cells, Molecules 20 (2015) 15343-15358; https://doi.org/10.3390/molecules200815343

5. M. Ambroz, P. Matouskova, A. Skarka, M. Zajdlova, K. Zakova and L. Skalova, The effects of selected sesquiterpenes from Myrica rubra essential oil on the efficacy of doxorubicin in sensitive and resistant cancer cell lines, Molecules 22 (2017) Article ID 1021 (10 pages); https://doi.org/10.3390/ molecules220610214

6. J. Legault and A. Pichette, Potentiating effect of beta-caryophyllene on anticancer activity of alpha-humulene, isocaryophyllene and paclitaxel, J. Pharm. Pharmacol. 59 (2007) 1643-1647; https:// doi.org/10.1211/jpp.59.12.0005

7. C. Kim, S. K. Cho, K. D. Kim, D. Nam, W. S. Chung, H. J. Jang, S. G. Lee, B. S. Shim, G. Sethi and K. S. Ahn, Beta-caryophyllene oxide potentiates TNF alpha-induced apoptosis and inhibits invasion through down-modulation of NF-kappa B-regulated gene products, Apoptosis 19 (2014) 708 718; https://doi.org/10.1007/s10495-013-0957-9

8. M. Ambroz, V. Hanusova, A. Skarka, I. Bousova, V. Kralova, L. Langhasova and L. Skalova, Essential oil from Myrica rubra leaves potentiated antiproliferative and prooxidative effect of doxorubicin and its accumulation in intestinal cancer cells, Planta Med. 82 (2016) 89-96; https://doi. org/10.1055/s-0035-1558083

9. M. Li, L.-H. Song, G. G.-L. Yue, J. K.-M. Lee, L.-M. Zhao, L. Li, X. Zhou, S. K.-W. Tsui, S. S.-M. Ng, K.-P. Fung, N.-H. Tan and C. B.-S. Lau, Bigelovin triggered apoptosis in colorectal cancer in vitro and in vivo via upregulating death receptor 5 and reactive oxidative species, Sci. Rep. 7 (2017) Article ID 42176 (13 pages); https://doi.org/10.1038/srep42176

10. A. Rasul, R. Bao, M. Malhi, B. Zhao, I. Tsuji, J. Li and X. M. Li, Induction of apoptosis by costunolide in bladder cancer cells is mediated through ROS generation and mitochondrial dysfunction, Molecules 18 (2013) 1418-1433; https://doi.org/10.3390/molecules18021418

11. Y. S. Sun, L. X. Lv, Z. Zhao, X. He, L. You, J. K. Liu and Y. Q. Li, Cordycepol C induces caspaseindependent apoptosis in human hepatocellular carcinoma HepG2 cells, Biol. Pharm. Bull. 37 (2014) 608-617.

12. T. C. Chou, Drug combination studies and their synergy quantification using the Chou-Talalay method, Cancer Res. 70 (2010) 440-446; https://doi.org/10.1158/0008-5472.CAN-09-1947

13. B. B. Zheng, L. H. Wu, L. S. Ma, S. S. Liu, L. Li, W. D. Xie and X. Li, Telekin induces apoptosis associated with the mitochondria-mediated pathway in human hepatocellular carcinoma cells, Biol. Pharm. Bull. 36 (2013) 1118-1125. 